\title{
Feasibility and antitumor efficacy in vivo, of simultaneously targeting glycolysis, glutaminolysis and fatty acid synthesis using lonidamine, 6-diazo-5-oxo-L-norleucine and orlistat in colon cancer
}

\author{
DIANA CERVANTES-MADRID ${ }^{1 *}$, GUADALUPE DOMINGUEZ-GOMEZ ${ }^{1 *}$, \\ AURORA GONZALEZ-FIERRO ${ }^{1}$, ENRIQUE PEREZ-CARDENAS ${ }^{1}$, LUCIA TAJA-CHAYEB ${ }^{1}$, \\ CATALINA TREJO-BECERRIL ${ }^{1}$ and ALFONSO DUENAS-GONZALEZ ${ }^{2,3}$
}

${ }^{1}$ Division of Basic Research, National Cancer Institute; ${ }^{2}$ Unit of Biomedical Research on Cancer, Institute of Biomedical Investigations, National Autonomous University of Mexico/National Cancer Institute, Mexico City 14080; ${ }^{3}$ Unit of Basic and Applied Research, ISSEMyM Cancer Center, Toluca 50180, Mexico

Received May 19, 2016; Accepted September 13, 2016

DOI: $10.3892 / \mathrm{ol} .2017 .5615$

\begin{abstract}
The aim of the present study was to investigate in vivo the feasibility and efficacy of the combination of lonidamine (LND), 6-diazo-5-oxo-L-norleucine (DON) and orlistat to simultaneously target glycolysis, glutaminolysis and de novo synthesis of fatty acids, respectively. The doses of LND and DON used in humans were translated to mouse doses $(77.7 \mathrm{mg} / \mathrm{kg}$ and $145.5 \mathrm{mg} / \mathrm{kg}$, respectively) and orlistat was used at $240 \mathrm{mg} / \mathrm{kg}$. Three schedules of LND, DON and orlistat at different doses were administered by intraperitoneal injection to BALB/c mice in a 21-day cycle (schedule 1: LND, $0.5 \mathrm{mg} /$ day; DON, $0.25 \mathrm{mg} /$ day 1, 5 and 9; orlistat, $240 \mathrm{mg} / \mathrm{kg} /$ day; schedule 2: LND, $0.1 \mathrm{mg} /$ day; DON, $0.5 \mathrm{mg} /$ day 1,5 and 9 ; orlistat, $240 \mathrm{mg} / \mathrm{kg} /$ day; schedule 3 : LND, $0.5 \mathrm{mg} /$ day; DON, $0.08 \mathrm{mg} /$ day 1, 5 and 9; orlistat, $360 \mathrm{mg} / \mathrm{kg} /$ day) to assess tolerability. To determine the antitumor efficacy, a syngeneic tumor model in BALB/c mice was created using colon cancer CT26.WT cells, and a xenogeneic tumor model was created in nude mice using the human colon cancer SW480 cell line. Mice were treated with schedule 1. Animals were weighed, clinically inspected during the experiment and the tumor volume was measured at day 21 .
\end{abstract}

Correspondence to: Dr Alfonso Duenas-Gonzalez, Unit of Biomedical Research on Cancer, Institute of Biomedical Investigations, National Autonomous University of Mexico/National Cancer Institute, 22 San Fernando, Mexico City 14080, Mexico

E-mail: alfonso_duenasg@yahoo.com

*Contributed equally

Key words: cancer metabolism, glycolysis, glutaminolysis, de novo synthesis of fatty acids, lonidamine, 6-diazo-5-oxo-L-norleucine, orlistat
The 3 schedules assessed in the tolerability experiments were well tolerated, as mice maintained their weight and no evident clinical signs of toxicity were observed. Combination treatment with schedule 1 significantly decreased tumor growth in each mouse model. No evident signs of toxicity were observed and mice maintained their weight during treatment. The triple metabolic blockade of the malignant phenotype appears feasible and promising for cancer therapy.

\section{Introduction}

Cancer cells commonly exhibit a malignant metabolic phenotype, which is characterized by increased rates of glycolysis, glutaminolysis and de novo synthesis of fatty acids (FAs) compared with normal cells. These metabolic alterations result from diverse gain-of-function mutations in oncogenes and loss-of-function of tumor suppressor genes, which aid cancer cells to thrive under various environmental conditions (1).

Glucose and glutamine supply the majority of the necessary carbon and nitrogen for the synthesis of macromolecules, energy and reducing equivalents to support cell growth through glycolysis and glutaminolysis (2). Lipogenesis is a third metabolic feature of cancer. In general, malignant cells synthetize de novo FAs instead of taking them up from the circulation, and malignant cells frequently overexpress FA synthase (FASN) (3). For de novo synthesis of FAs, glucose and glutamine supply citrate. Glucose is converted to acetyl-coenzyme A (CoA) in the mitochondrial matrix to synthesize citrate in the tricarboxylic acid (TCA) cycle, whereas glutamine supplies carbon in the form of mitochondrial oxaloacetate to maintain citrate production in the first step of the TCA cycle (4). Thus, the metabolism of glutamine and glucose is orchestrated to support the production of acetyl-CoA and NADPH required for fatty acid synthesis (4).

Despite the strong rationale for developing a combination of drugs to simultaneously target these three key processes in malignant cells, to the best of our knowledge, there is 
no preclinical in vivo evidence supporting the antitumor activity of this triple targeting, despite the availability of well-characterized pharmacological inhibitors of these enzymes (5). Among anti-glycolytic and anti-glutaminolytic drugs, lonidamine (LND) and 6-diazo-5-oxo-L-norleucine (DON) are well-known inhibitors of hexokinase II (HK-II) and glutaminase $\mathrm{K}$, respectively, which have previously been clinically evaluated (5). Regarding lipogenesis, a number of experimental compounds have been developed; however, none have reached clinical trials (6). Among these, orlistat has shown promising activity in a number of malignancies due to its ability to inhibit FASN, which is responsible for the de novo synthesis of FA $(7,8)$. It was previously reported that LND, DON and orlistat inhibit cell viability in a number of human cancer cell lines, and that these drugs are synergistic in vitro (9). The present study demonstrated that this triple combination is feasible and effective against tumor models in mice.

\section{Materials and methods}

Cell lines, drugs and preparations. Human colon cancer SW480 and mouse colon cancer CT26.WT cell lines were obtained from the American Type Culture Collection (Manassas, VA, USA) and cultured in DMEM-F15 and RPMI-1640 respectively, supplemented with $10 \%$ fetal bovine serum (Gibco; Thermo Fisher Scientific, Inc., Waltham, MA, USA), in a humidified $5 \% \mathrm{CO}_{2}$ atmosphere at $37^{\circ} \mathrm{C}$. LND, DON and orlistat were purchased from Sigma-Aldrich (Merck Millipore, Darmstadt, Germany). Stock solutions were prepared using dimethyl sulfoxide, water and ethanol for LND $(11 \mathrm{mg} / \mathrm{ml})$, DON $(16 \mathrm{mg} / \mathrm{ml})$ and orlistat $(200 \mathrm{mg} / \mathrm{ml})$ respectively.

Doses. To investigate whether the combined administration of these drugs was clinically feasible and following assessment of existing pharmacokinetic data in clinical studies of LND and DON (5), the human doses of LND and DON used in clinical trials were translated to mouse doses using the formula: Mouse equivalent dose=human dose $(\mathrm{mg} / \mathrm{kg})$ x human $\mathrm{K}_{\mathrm{m}} /$ mouse $_{\mathrm{m}}$, where the human and mouse $\mathrm{K}_{\mathrm{m}}$ was 37 and 3, respectively, as reported by Reagan-Shaw et al (10). As shown in Table I, the human doses of LND and DON, each used as a single agent, were as follows: $450 \mathrm{mg} /$ daily $(6.3 \mathrm{mg} / \mathrm{kg}$ day assuming a $70 \mathrm{~kg}$ patient) for LND and a $480 \mathrm{mg} / \mathrm{m}^{2}$ DON total dose (divided between days 1, 2 and 3). DON is prescribed in humans by $\mathrm{m}^{2}$; therefore this dose was first converted to $\mathrm{mg} / \mathrm{kg}$ by assuming $1.7 \mathrm{~m}^{2}$ of body surface area, which results in $825 \mathrm{mg} / 70 \mathrm{~kg}=11.8 \mathrm{mg} / \mathrm{kg}$. Thus, using these human doses to calculate the doses for mice weighting $20 \mathrm{~g}$, three different schedules were administered (Table I). For orlistat, which is used systemically in cancer models, there exists only preclinical information, and in the majority of cases it is used at $240 \mathrm{mg} / \mathrm{m}^{2}$ in mice $(7,8)$. Therefore, this dose was used in schedules 1 and 2, but a dose of $360 \mathrm{mg} / \mathrm{m}^{2}$ was used in schedule 3 to gain insight into its tolerability beyond common doses used in mice.

Tolerability of the triple combination in vivo. To study these schedules of the triple combination that are tolerable when injected into healthy mice, groups of 6 -week-old BALB/c female mice (6 mice per group, 36 mice in total; Harlan Laboratories, Mexico City, Mexico) were treated with the triple combination of LND, DON and orlistat for a 21-day cycle. Mice were allowed to acclimatize for 1 week and kept in a 12:12 light-dark cycle with access to food and water ad libitum. The three treatment schedules are shown in Table I. The three drugs were intraperitoneally administered, with at least $3 \mathrm{~h}$ between each injection and careful skin disinfection to avoid infectious peritonitis. The total volume of injection was $<20 \mu 1$ for each drug. The control group was injected with the vehicle of each drug, at identical volumes to the treatment groups. Mice were weighed and clinically inspected on days $0,5,9,15$ and 19 .

Antitumor effect of the triple combination in vivo in a syngeneic model. Six week-old BALB/c female mice (6 mice per group, 12 in total) were obtained from Harlan Laboratories. Mice were allowed to acclimatize for 1 week prior to starting the experiments. Housing conditions included access to food and water ad libitum in a 12:12 light-dark cycle. Handling was performed inside a laminar flow cabinet, and a total of $4 \times 10^{5}$ CT26.WT cells were injected in one flank. The treatment commenced 2 weeks subsequent to inoculation, when the tumors measured $\sim 100 \mathrm{~mm}^{3}$. The treatment consisted of intraperitoneal injection of $0.5 \mathrm{mg}$ LND daily (total dose), $0.25 \mathrm{mg}$ DON on days 1 , 5 and 9 (total dose, $0.75 \mathrm{mg}$ ), and $240 \mathrm{mg} / \mathrm{kg}$ orlistat daily, which constituted schedule 1 of the tolerability experiment, in a cycle of 21 days. Drug administration was performed as aforementioned. Animals were weighed, clinically inspected and tumors were measured with electronic calipers, and the tumor volume was estimated using the formula $\mathrm{axb}^{2} \mathrm{x}(\pi / 6)=\mathrm{V}$ $\left(\mathrm{mm}^{3}\right)$, where $\mathrm{a}$ is the major diameter, $\mathrm{b}$ is the minor diameter and $\mathrm{V}$ is the volume. At the end of treatment the mice were sacrificed in a $\mathrm{CO}_{2}$ chamber and necropsied. Tumors were dissected and weighed. Visual inspection of major organs was performed.

Antitumor effect of the triple combination in an allogeneic model. Two groups six-week-old BALB/c nu/nu female mice, with 6 mice per group, were obtained from Harlan Laboratories. Acclimatization, housing, feeding and manipulation were performed as aforementioned. Nude mice were injected with $1 \times 10^{6} \mathrm{SW} 480$ cells in each flank (total dose, $2 \times 10^{6}$ cells). The treatment was started 2 weeks subsequent to inoculation, when the tumors were $\sim 250 \mathrm{~mm}^{3}$ in size. The treatment consisted of intraperitoneal injection of $0.5 \mathrm{mg}$ LND daily (total dose), $0.25 \mathrm{mg}$ DON on days 1, 5 and 9 (total dose, $0.75 \mathrm{mg}$ ) and $240 \mathrm{mg} / \mathrm{kg}$ orlistat daily in a 21-day cycle, which constituted schedule 1 of the tolerability experiment. Drug administration was performed as aforementioned. Animals were weighed, clinically inspected and tumors were measured with electronic calipers, and the tumor volume was estimated using the formula $\mathrm{axb}^{2} \mathrm{x}(\pi / 6)=\mathrm{V}\left(\mathrm{mm}^{3}\right)$, where $\mathrm{a}$ is the major diameter, $\mathrm{b}$ is the minor diameter and $\mathrm{V}$ is the volume. At the end of treatment the mice were sacrificed in a $\mathrm{CO}_{2}$ chamber and necropsied. Tumors were dissected and weighed. Visual inspection of major organs was performed.

Ethics statement. All animal studies were designed to reduce the suffering of the animals, and were performed in 
Table I. Dose schedule of LND, DON and orlistat used in mice.

\begin{tabular}{|c|c|c|c|c|}
\hline Drug & Schedule 1 & Schedule 2 & Schedule 3 & Human dose \\
\hline LND & $25 \mathrm{mg} / \mathrm{kg}, 0.5 \mathrm{mg} /$ day & $5 \mathrm{mg} / \mathrm{kg}, 0.1 \mathrm{mg} / \mathrm{day}$ & $25 \mathrm{mg} / \mathrm{kg}, 0.5 \mathrm{mg} / \mathrm{day}$ & $6.3 \mathrm{mg} / \mathrm{kg}, 450 \mathrm{mg} /$ day \\
\hline DON & $\begin{array}{l}36.2 \mathrm{mg} / \mathrm{kg}, 0.75 \mathrm{mg} \text { divided } \\
\text { between days } 1,5 \text { and } 9\end{array}$ & $\begin{array}{l}72.5 \mathrm{mg} / \mathrm{kg}, 1.5 \mathrm{mg} \text { divided } \\
\text { between days } 1,5 \text { and } 9\end{array}$ & $\begin{array}{l}12.6 \mathrm{mg} / \mathrm{kg}, 0.25 \mathrm{mg} \text { divided } \\
\text { between days } 1,5 \text { and } 9\end{array}$ & $\begin{array}{l}11.8 \mathrm{mg} / \mathrm{kg} \text { divided } \\
\text { between days } 1,2 \text { and } 3\end{array}$ \\
\hline Orlistat & $240 \mathrm{mg} / \mathrm{kg}$ daily & $240 \mathrm{mg} / \mathrm{kg}$ daily & $360 \mathrm{mg} / \mathrm{kg}$ daily & Unknown \\
\hline
\end{tabular}

The dose translation between humans and mice was calculated using the formula by Reagan-Shaw et al (10). A fixed weight of $20 \mathrm{~g}$ for mice and $70 \mathrm{~kg}$ for humans was used for calculations. DON is prescribed in human by $\mathrm{m}^{2}$, therefore it was first converted to $\mathrm{mg} / \mathrm{kg}$, assuming $1.7 \mathrm{~m}^{2}$ of body surface area, which resulted in $825 \mathrm{mg} / 70 \mathrm{~kg}=11.8 \mathrm{mg} / \mathrm{kg}$. LND, lonidamine; DON, 6-diazo-5-oxo-L-norleucine.

compliance with the policies of the Institutional Research Ethics Board and Animal Care Committee of the Instituto Nacional de Cancerología (Mexico City, Mexico) (permit numbers, CA006/CB595/10 and INCAN/CC/010/10).

Statistical analysis. Data are presented as the mean \pm standard deviation. Statistical differences in weight among the groups of mice treated with different schedules were evaluated using analysis of variance, and the tumor volumes at each time and the final weight of the tumors between the control and treated groups were evaluated with paired Student's $t$-test. Statistical analyses were conducted using SPSS (SPSS, Inc., Chicago, IL, USA).

\section{Results}

Tolerability in vivo. Previous results from our laboratory (9) showed that in vitro treatment with the combination of LND, DON and orlistat is highly synergistic and has increased antitumor effects compared with treatment with each drug alone. Additionally, it was found that total doses of $0.25 \mathrm{mg}$ LND and $0.25 \mathrm{mg}$ DON plus $240 \mathrm{mg} / \mathrm{m}^{2}$ orlistat are well tolerated in mice. To confirm these results, additional doses were tested using the human equivalent dose in mice as a reference, as shown in Table I. The three schedules tested were shown to be well tolerated. There was a transient decrease in weight during the first 9 days of treatment, but weight was recuperated by day 19 . No statistically significant differences were found $(\mathrm{P}=0.788$; Fig. 1). Mice showed no hair frizzing or hypoactivity. No other clinical signs of toxicity were observed.

Antitumor effects in the syngeneic model. Based on these results, schedule 1 was chosen (total doses of $0.5 \mathrm{mg}$ LND daily and $0.75 \mathrm{mg}$ DON divided over three days). The treatment was well tolerated and tumor volumes $(\mathrm{P}=0.0455)$ and tumor weights $(\mathrm{P}=0.0005)$ were significantly lower in the treated animals; however, animals in the control group showed marked hypoactivity, hair frizzing and weight loss after day 10. Therefore, animals were sacrificed at day 14 (Fig. 2A and B).

Antitumor effects in the allogeneic model. Treatment with schedule 1 was also well tolerated in the nude mice injected with the human colon cancer SW480 cell line. Total weight was not significantly different between the two groups, and no evident signs of toxicity were noted. However, tumor volumes

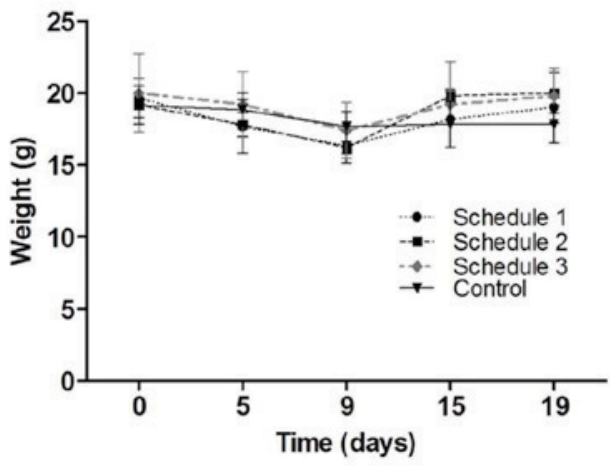

Figure 1. Tolerability of three schedules of lonidamine, 6-diazo-5-oxo-L-norleucine and orlistat administration in BALB/c mice. Groups of 6 mice were treated with schedules 1, 2 and 3, as shown in Table I, by intraperitoneal injection. The control group received vehicle treatment only. The mice had no significant differences in weight loss between the groups $(\mathrm{P}=0.788)$ and no signs of toxicity were exhibited.

were $>2$-fold lower in the treated animals and showed extensive areas of necrosis. As shown in Fig. 3A and B, curves of volume began to separate between day 4 and the end of treatment $(\mathrm{P}=0.0351)$, and the tumor weights were significantly decreased in the treated animals $(\mathrm{P}=0.0002)$.

\section{Discussion}

The results of the present study show that the systemic administration of a pharmacological combination of inhibitors of glycolysis, glutaminolysis and the de novo synthesis of FAs is not only well tolerated, as demonstrated by no changes in body weight and no evident signs of toxicity, but that exerts antitumor effects in syngeneic mice injected with a murine colon carcinoma and in nude mice bearing human colon carcinoma cells.

The three most common, or at least most studied, metabolic alterations of cancer cells are glycolysis, glutaminolysis and the de novo synthesis of FAs (1-3). The increased activities of these pathways are therefore natural targets to attack the malignant metabolic phenotype. However, antitumor strategies targeting the malignant metabolic phenotype attempt to target these processes separately (11-13).

A number of preclinical studies using drugs to target these pathways demonstrate that they are effective (14-17). Among glycolytic inhibitors, a number of drugs are being evaluated in 
A

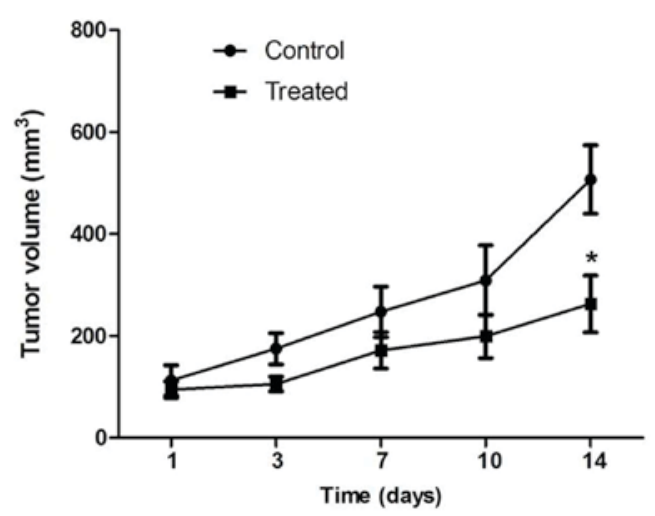

B

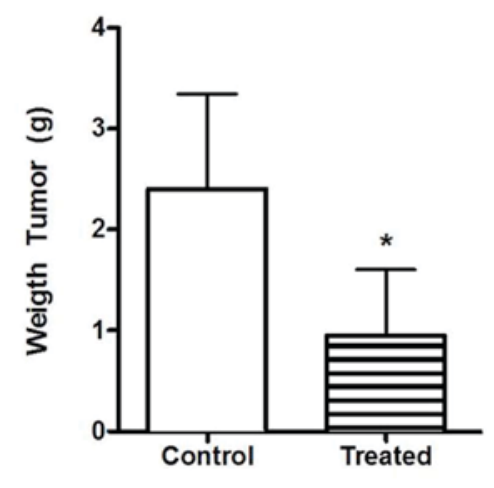

Figure 2. Antitumor effects of the combination treatment in the syngeneic mouse model. (A) Mice (6 per group) were treated with schedule 1, consisting of $0.5 \mathrm{mg}$ lonidamine daily, $0.25 \mathrm{mg}$ 6-diazo-5-oxo-L-norleucine on days 1,5 and 9 (0.75 mg/total) and $240 \mathrm{mg} / \mathrm{kg}$ orlistat. The control group received vehicle treatment only. Treated mice had significantly lower tumor volumes, as shown on the growth curves $(\mathrm{P}=0.0455)$, (B) Significant differences in tumor weights were also observed $(\mathrm{P}=0.0005)$. The experiment was terminated on day 14 as control mice had experienced marked hypoactivity, hair frizzing and weight loss since day $10 .{ }^{*} \mathrm{P}<0.05$.

A

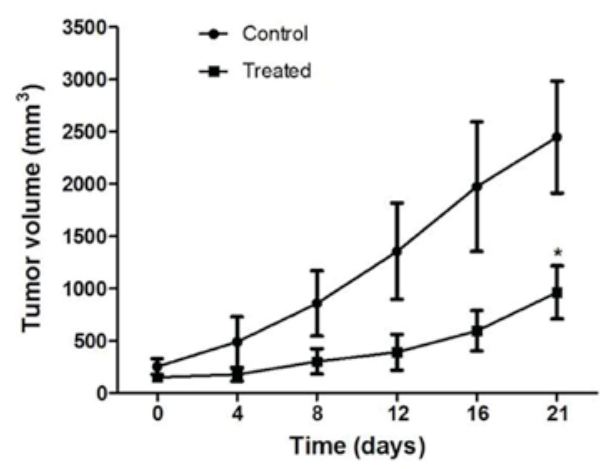

B

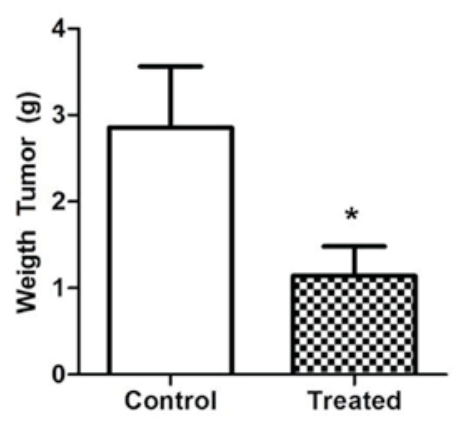

Figure 3. Antitumor effects of the combination treatment in the allogeneic mouse model. (A) In total, 6 nude mice per group were treated with schedule 1 , consisting of $0.5 \mathrm{mg}$ lonidamine daily, $0.25 \mathrm{mg} \mathrm{6-diazo-5-oxo-L-norleucine} \mathrm{on} \mathrm{days} 1,5 \mathrm{and} 9$ ( $0.75 \mathrm{mg}$ total), and $240 \mathrm{mg} / \mathrm{kg}$ orlistat. The control group received vehicle treatment only. Tumor growth was significantly reduced in treated mice $(\mathrm{P}=0.0351)$. (B) Significantly decreased weight of tumors was observed in treated mice $(\mathrm{P}=0.0002) .{ }^{*} \mathrm{P}<0.05$.

experimental systems, as reviewed by Ganapathy-Kanniappan and Geschwind (14). However, only LND, 2-deoxy-D-glucose and dichloracetate have reached clinical trials, with modest results as single agents or in combination with chemotherapy or radiation (15-17). In particular, LND, the HK-II inhibitor used in the present study has been widely investigated for the treatment of solid tumors with encouraging results in phase II-III trials for the treatment of advanced breast, ovarian and lung cancer (15). Regarding glutaminolysis inhibitors, the 3 diazo analogs of L-glutamine, azaserine, DON and azotomycin, showed clinical antitumor activity (18), but have not been further studied, with the exception of DON, which was used with recombinant glutaminase and showed promising results (19). Newer selective agents against glutaminase are being developed. One of these new agents, CB-839, has recently entered into clinical trials (ClinicalTrial.gov identifiers NCT02071862, NCT02071888 and NCT02071927). No clinical trials in cancer have been undertaken with FASN inhibitors. Among FASN inhibitors, orlistat shows promising activity in vitro and in vivo in a number of malignancies due to its ability to inhibit FASN, which is responsible for the de novo synthesis of FAs (8).

To the best of our knowledge, no preclinical studies have been performed using a drug combination concurrently targeting these 3 metabolic alterations beyond our previous study (9). The most similar study was reported in 1993, in which the combination of DON and 2-deoxy-D-glucose led to marked inhibition of glutamine oxidation and glycolysis, which was accompanied by increased cytotoxicity against the human myeloid TPH-1 cell line and freshly cultured myeloid blast cultures obtained from a patient (20). Thus, the results of the present study support the hypothesis that the pharmacological blockade of the three main metabolic pathways is feasible and exhibits antitumor activity.

The results of the present study regarding the doses and schedule used may be observed as an approximation only. In regard to orlistat, no preclinical pharmacokinetic studies have been reported. Effective antitumor doses in vitro are between 
25 and $100 \mu \mathrm{M}(8)$ and when used in mice, the most frequently administered dose is $240 \mathrm{mg} / \mathrm{kg}$. Kridel et al (7) reported peak blood levels of orlistat to be $\sim 10 \mu \mathrm{M} 2 \mathrm{~h}$ subsequent to a single intraperitoneal administration at $155 \mathrm{mg} / \mathrm{kg}$ in mice (7). These results suggest that at $240 \mathrm{mg} / \mathrm{kg}$, therapeutic effective dose could be achieved in plasma; however, this must be confirmed by pharmacokinetic studies. Notably, new formulations of micellar nanoparticles of orlistat for cancer treatment are in development (21), as the systemic levels of orlistat used orally for obesity are $<10 \mathrm{ng} / \mathrm{ml}(0.02 \mu \mathrm{M})$ due to its poor absorption (22). For determining the dose of LND and DON used in mice, the human dose (10) was translated to a mouse dose, which is also an approximation. However, pharmacokinetic analyses of the 3 drugs should be performed to corroborate the appropriateness of the doses and to determine potential pharmacokinetic interactions among them. It should be noted that the doses used here for LND and DON are well below those used in published preclinical trials (5). Thus, in the 3 schedules, doses of 25,5 and $25 \mathrm{mg} / \mathrm{kg}$ LND were used compared with 50 and $100 \mathrm{mg} / \mathrm{kg}$ in the literature $(23,24)$. Similarly, doses of 36.2 , 72.5 and $12.6 \mathrm{mg} / \mathrm{kg}$ DON were used, whereas in the literature the mean dose is $15.03 \mathrm{mg} / \mathrm{kg}$ (range, $0.02-100 \mathrm{mg} / \mathrm{kg}$ ), with a mean of 13 days (range, 9-28 days) of administration in a 28-day cycle (25). The present data suggest that the strong synergy observed in vitro at drug concentrations well below those used separately (9) could also occur in vivo. It also should be noted that the combination was effective in the syngeneic (and low tumor burden) and allogeneic (and high tumor burden) groups, suggesting that this treatment is effective in murine and human colon carcinomas, as well as in low and high tumor burdens. This was not unexpected, since metabolic reprogramming in the tumor use of glucose, glutamine and FAs, to different extents, is a common feature of cancer cells.

In summary, the present results support the hypothesis that targeting cancer metabolism by simultaneously inhibiting 3 key metabolic pathways may actually have a wide therapeutic window (26), as no unacceptable effects in mice were observed when treated at translated doses slightly below to those used in patients for LND and DON administered separately. Notably, DON and LND are drugs that are currently being re-studied (27-29), while formulations of orlistat suitable for systemic administration have been investigated (19). Thus, triple pharmacological metabolic blockade of the malignant phenotype appears feasible and promising for cancer therapy. However, despite the target inhibition of the three drugs used here being strongly demonstrated in other preclinical models $(5,6)$, additional studies are required to confirm whether the triple metabolic blockade with this drug combination changes the rate of oxidation of glucose, glutamine and fatty acids in tumors.

\section{Acknowledgements}

The present study was supported by CONACyT (grant nos. 140654 and SB0771).

\section{References}

1. Chen JQ and Russo J: Dysregulation of glucose transport, glycolysis, TCA cycle and glutaminolysis by oncogenes and tumor suppressors in cancer cells. Biochim Biophys Acta 1826: 370-384, 2012
2. Moncada S, Higgs EA and Colombo SL: Fulfilling the metabolic requirements for cell proliferation. Biochem J 446: 1-7, 2012.

3. Menendez JA and Lupu R: Fatty acid synthase and the lipogenic phenotype in cancer pathogenesis. Nat Rev Cancer 7: 763-767, 2007.

4. Tan NL and Seyfried TN: Influence of serum and hypoxia on incorporation of [(14)C]-D-glucose or [(14)C]-L-glutamine into lipids and lactate in murine glioblastoma cells. Lipids 50: 1167-1184, 2015.

5. Cervantes-Madrid D, Romero Y and Dueñas-González A: Reviving lonidamine and 6-Diazo-5-oxo-L-norleucine to be used in combination for metabolic cancer therapy. Biomed Res Int 2015: 690492, 2015.

6. Lupu R and Menendez JA: Pharmacological inhibitors of fatty acid synthase (FASN)-catalyzed endogenous fatty acid biogenesis: A new family of anti-cancer agents? Curr Pharm Biotechnol 7: 483-493, 2006.

7. Kridel SJ, Axelrod F, Rozenkrantz N and Smith JW: Orlistat is a novel inhibitor of fatty acid synthase with antitumor activity. Cancer Res 64: 2070-2075, 2004.

8. Flavin R, Peluso S, Nguyen PL and Loda M: Fatty acid synthase as a potential therapeutic target in cancer. Future Oncol 6: 551-562, 2010.

9. Cervantes-Madrid D and Dueñas-González A: Antitumor effects of a drug combination targeting glycolysis, glutaminolysis and de novo synthesis of fatty acids. Oncol Rep 34: 1533-1542, 2015.

10. Reagan-Shaw S, Nihal M and Ahmad N: Dose translation from animal to human studies revisited. FASEB J 22: 659-661, 2008.

11. Elf SE and Chen J: Targeting glucose metabolism in patients with cancer. Cancer 120: 774-780, 2014.

12. Jin L, Alesi GN and Kang S: Glutaminolysis as a target for cancer therapy. Oncogene 35:3619-3625, 2016.

13. Deepa PR, Vandhana S, Jayanthi U and Krishnakumar S: Therapeutic and toxicologic evaluation of anti-lipogenic agents in cancer cells compared with non-neoplastic cells. Basic Clin Pharmacol Toxicol 110: 494-503, 2012.

14. Ganapathy-Kanniappan S and Geschwind JF: Tumor glycolysis as a target for cancer therapy: Progress and prospects. Mol Cancer 12: 152, 2013.

15. Di Cosimo S, Ferretti G, Papaldo P, Carlini P, Fabi A and Cognetti F: Lonidamine: Efficacy and safety in clinical trials for the treatment of solid tumors. Drugs Today (Barc) 39: 157-174, 2003.

16. Dwarakanath BS, Singh D, Banerji AK, Sarin R, Venkataramana NK, Jalali R, Vishwanath PN, Mohanti BK, Tripathi RP, Kalia VK and Jain V: Clinical studies for improving radiotherapy with 2-deoxy-D-glucose: Present status and future prospects. J Cancer Res Ther 5: (Suppl 1) S21-S26, 2009.

17. Garon EB, Christ ofk HR, Hosmer W, Britten CD, Bahng A, Crabtree MJ, Hong CS, Kamranpour N, Pitts S, Kabbinavar F, et al: Dichloroacetate should be considered with platinum-based chemotherapy in hypoxic tumors rather than as a single agent in advanced non-small cell lung cancer. J Cancer Res Clin Oncol 140: 443-452, 2014.

18. Catane R, Von Hoff DD, Glaubiger DL and Muggia FM: Azaserine, DON, and azotomycin: Three diazo analogs of L-glutamine with clinical antitumor activity. Cancer Treat Rep 63: 1033-1038, 1979.

19. Unger C, Mueller C, Bausch MP, Krzemieniecki K, Ochenduszko S, Wilk B, Jaeger E and Al-Batran S: A phase I schedule optimization study of pegylated glutaminase (PEG-PGA) plus 6-diazo-5-oxo-1-norleucine (DON) in patients (pts) with advanced solid tumors. J Clin Oncol 29: (Suppl) abstr 3049 , 2011.

20. Griffiths M, Keast D, Patrick G, Crawford M and Palmer TN: The role of glutamine and glucose analogues in metabolic inhibition of human myeloid leukaemia in vitro. Int J Biochem 25: 1749-1755, 1993.

21. Paulmurugan R, Bhethanabotla R, Mishra K, Devulapally R, Foygel K, Sekar TV, Ananta JS, Massoud TF and Joy A: Folate receptor targeted polymeric micellar nanocarriers for delivery of orlistat as a repurposed drug against triple negative breast cancer. Mol Cancer Ther 15: 221-231, 2016.

22. Zhi J, Mulligan TE and Hauptman JB: Long-term systemic exposure of orlistat, a lipase inhibitor, and its metabolites in obese patients. J Clin Pharmacol 39: 41-46, 1999.

23. De Cesare M, Pratesi G, Giusti A, Polizzi D and Zunino F: Stimulation of the apoptotic response as a basis for the therapeutic synergism of lonidamine and cisplatin in combination in human tumour xenografts. Br J Cancer 77: 434-439, 1998. 
24. Nath K, Nelson DS, Heitjan DF, Leeper DB, Zhou R and Glickson JD: Lonidamine induces intracellular tumor acidification and ATP depletion in breast, prostate and ovarian cancer xenografts and potentiates response to doxorubicin. NMR Biomed 28: 281-290, 2015.

25. Ovejera AA, Houchens DP, Catane R, Sheridan MA and Muggia FM: Efficacy of 6-diazo-5-oxo-L-norleucine and $\mathrm{N}$-(N-gamma-glutamyl-6-diazo-5-oxo-norleucinyl)-6

-diazo-5- oxo-norleucine against experimental tumors in conventional and nude mice. Cancer Res 39: 3220-3224, 1979.

26. Vander Heiden MG: Targeting cancer metabolism: A therapeutic window opens. Nat Rev Drug Discov 10: 671-684, 2011.
27. Guo L, Shestov AA, Worth AJ, Nath K, Nelson DS, Leeper DB, Glickson JD and Blair IA: Inhibition of mitochondrial complex II by the anti-cancer agent lonidamine. J Biol Chem 291: 42-57, 2016.

28. Assanhou AG, Li W, Zhang L, Xue L, Kong L, Sun H, Mo R and Zhang C: Reversal of multidrug resistance by co-delivery of paclitaxel and lonidamine using a TPGS and hyaluronic acid dual-functionalized liposome for cancer treatment. Biomaterials 73: 284-295, 2015.

29. Olsen RR, Mary-Sinclair MN, Yin Z and Freeman KW: Antagonizing Bcl-2 family members sensitizes neuroblastoma and Ewing's sarcoma to an inhibitor of glutamine metabolism. PLoS One 10: e0116998, 2015. 